\title{
THE ACOUSTIC, THE DIGITAL AND THE BODY: A SURVEY ON MUSICAL INSTRUMENTS
}

\author{
Thor Magnusson \\ Creative Systems Lab \\ University of Sussex \\ Brighton, United Kingdom \\ +44 (0) 1273678195 \\ thor@ixi-software.net
}

\author{
Enrike Hurtado Mendieta \\ Digital Research Unit \\ University of Huddersfield \\ Huddersfield, United Kingdom \\ +44 (0) 1484473287 \\ enrike@ixi-software.net
}

\begin{abstract}
This paper reports on a survey conducted in the autumn of 2006 with the objective to understand people's relationship to their musical tools. The survey focused on the question of embodiment and its different modalities in the fields of acoustic and digital instruments. The questions of control, instrumental entropy, limitations and creativity were addressed in relation to people's activities of playing, creating or modifying their instruments. The approach used in the survey was phenomenological, i.e. we were concerned with the experience of playing, composing for and designing digital or acoustic instruments. At the time of analysis, we had 209 replies from musicians, composers, engineers, designers, artists and others interested in this topic. The survey was mainly aimed at instrumentalists and people who create their own instruments or compositions in flexible audio programming environments such as SuperCollider, Pure Data, ChucK, Max/MSP, CSound, etc.
\end{abstract}

\section{Keywords}

Survey, musical instruments, usability, ergonomics, embodiment, mapping, affordances, constraints, instrumental entropy, audio programming.

\section{INTRODUCTION}

For more than six years we at ixi software [6][7] have been creating alternative instruments for the computer, focusing on the graphical user interface and its deterministic nature. We have tried to resist the temptation of imitating the world of acoustic instruments or physical hardware. The goal has been to create instruments that make effective use of the specific qualities of the computer itself with its various hardware interfaces, but concentrating particularly on the semiotic affordances of the screen as the main control interface.

We have created various instruments and software suites that are freely available on our website. We also run a mailing list, a net label and we give workshops in audio-visual programming at

Permission to make digital or hard copies of all or part of this work for personal or classroom use is granted without fee provided that copies are not made or distributed for profit or commercial advantage and that copies bear this notice and the full citation on the first page. To copy otherwise, or republish, to post on servers or to redistribute to lists, requires prior specific permission and/or a fee.

Nime'07, June 6-10, 2007, New York, New York, USA.

Copyright remains with the author(s). various universities and art institutions all over Europe. Although we have had good and instructive feedback from ixi software users, musical collaborators and workshop participants, we have been interested in developing more systematic feedback or dialog, which induced an interest to create a user survey addressing the questions that we are focusing on in our work.

In the survey we were specifically concerned with people's experience of the difference between playing an acoustic and a digital instrument. The approach was phenomenological and qualitative: we wanted to know how musicians or composers describe their practise and relationship with their musical tools, whether acoustic or digital. We deliberately did not define what we meant by "digital instrument" (such as sequencer software, graphical dataflow language, textual programming language or sensor interfaces mapped to sound) ${ }^{1}$, as we were interested in how people themselves define the digital, the acoustic, and the relationship between the two. How do people rate the distinctive affordances and constraints of these instruments and is there a difference in the way they critically respond to their design? Furthermore, do people relate differently to the makers of these two types of instruments? We were curious to learn if musical education and practise of an acoustic instrument yields a different critical relationship to the digital instrument. How does instrumental practice change the ideas of embodiment and does it affect the view of the qualitative properties of the computer-based tool? Finally, we were interested in knowing how people relate to the chaotic or "non-deterministic" nature of their instruments (if they see it as a limitation or a creative potential) and whether they feel that such "quality" could be arbitrarily ${ }^{2}$ designed into digital instruments.

In order to gain better understanding of these questions and the basis for people's views, we also asked the participants about their background in working with the computer; which operating systems they run; what software they predominantly use and the reasons they have chosen that environment for their work.

\footnotetext{
${ }^{1}$ For a discussion of the taxonomy of screen-based instruments or composing environments, see Duignan, Noble and Biddle [1]. Although the authors mainly focus on sequencing tools (and they acknowledge that they have a broad definition of the term) the taxonomy is still valid here in this context.

2 Arbitrarily, as everything has to be programmed into the digital instruments vs. the fact that acoustic instruments always contain those properties due to their materiality.
} 


\section{THE SURVEY}

\subsection{The Participants}

The group we tried to reach to with our survey was very wide, i.e. from the acoustic instrumentalist that has never touched a computer to the live-coder that does not play a traditional instrument. More specifically we were interested in learning about people that have a critical relationship with their tools (whether acoustic or digital) and build their own or modify existing instruments, to allow for their preferred way of expressing themselves musically. We were also curious to hear from people that have used our software how they experience ixi software in relation to the questions mentioned above.

The results we got were precisely from the group we focused on. The majority of the participants actively work with one or more of the audio programming environments that we asked about. There were very few replies from people that use exclusively commercial software such as Cubase, ProTools or Logic.

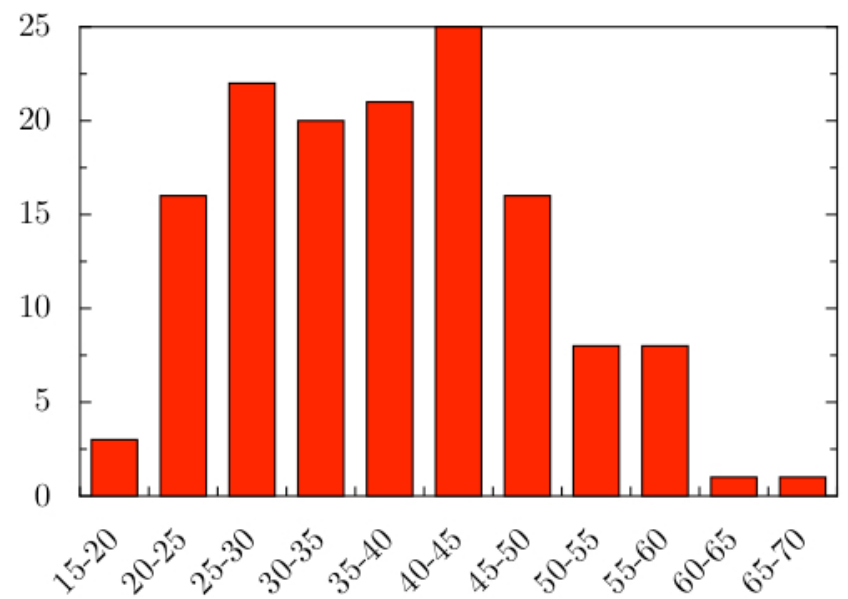

Figure 1. The age distribution of the survey participants. The $Y$ axis shows number of participants; $X$ axis the age-range.

To date, there have been 209 replies, mainly from Western Europe and North America, but a considerable amount also from South America and Asia. Of all the replies there were only 9 female participants, which is a frustrating state of affairs, but it is outside the scope of this survey and research to explore the reason behind this fact. However, we were interested in the age of the participants and how long they have been playing music. We were surprised how relatively high the mean age was (37 years), distributed as shown in Figure 1.

\subsection{The Questionnaire}

The survey is a qualitative survey where the main focus is on people's comments and description of their musical tools. We divided it into six areas:

1) Personal Details: a set of demographic questions on gender, age, profession, nationality, institutional affiliation, etc.;

2) Musical Background: questions on how the participants defined themselves in relation to the survey (musician, composer, designer, engineer, artist, other), how long they had played music, musical education, computer use in music and musical genre (if applicable);

3) Acoustic Instruments: questions about people's relationship with their instrument. (Which instrument, how long they have played it, etc.). We asked whether people found their instrument lacking in functionality; if they thought the instrument has "unstable" or "non-deterministic" behaviour; and if so, how they related to that. We asked how well they knew the history of their instrument and which factors affected the design of it. Would it be beneficial if the human body was different?

4) Digital Instruments: we asked which operating system people use and why; what hardware (computer, soundcard, controllers, sensors); what music software; and whether they have tried or use regularly the following audio programming environments: Pure Data, SuperCollider, ChucK, CSound, Max/MSP, Plogue Bidule, Aura, Open Sound World, AudioMulch and Reaktor. We asked about their programming experience and why they had chosen their software of choice. Further, we were interested in knowing if and how people use the Open Sound Control (OSC) and if people use programming environments for graphics or video in the context of their music making.

5) Comparison of Acoustic and Digital Instruments: here we were concerned with the difference of playing acoustic and digital instruments, and what each of the types lack or provide. We asked about people's dream software; what kind of interfaces people would like to use; and then if people found that the limitations of instruments are a source of frustration or inspiration. Did that depend on the type of instrument?

6) ixi software: this section of the questionnaire is only indirectly related to this paper. Here we wanted to know when and where the participants came across ixi software, how they use the software and which applications they use. We asked if there are characteristics in the design of the software that goes across the different applications and if these characteristics are signatures that influence the musical outcome. We were interested if people found they are free or limited in the use of ixi software. Is the graphical element (in the style that we use in ixi software) a positive or a negative feature?

People were free to answer the questions they were interested in and to skip the others, as it would not make sense to force an instrumentalist to answer questions about computers if he/she has never used one. The same goes for the audio programmer that does not play an acoustic instrument. For people interested in the questions, the survey resides here:

http://www.ixi-software.net/survey.

\subsection{The Methodology}

The survey was introduced on our website and we posted it to the ixi mailing list, but we also sent it to various external mailing lists (including SuperCollider, ChucK, Pure Data, Max/MSP, CSound, AudioMulch, eu-gene, livecode) and asked friends and collaborators to distribute the survey as much as possible. We contacted orchestras and conservatories and asked them to post the survey on their internal mailing lists. The survey could be answered in 9 languages, but the questions themselves were only available in English or Spanish. Unfortunately, as ca. 25 percent of the visitors on our website are from Japan where there is a strong culture of using audio programming languages, we did not have the resources to translate the survey into Japanese.

After 3 months of receiving replies, we started working on the data. We analysed each reply and put it into a database. All the quantitative data was filled in quickly, but as the questionnaire was largely qualitative (where people write their answers in the 
form of descriptive narrative), we had to interpret some of the data subjectively. Here we created a bipolar continuum (marked from 1 to 5) where the following "archetypal" elements were extracted: a) abstract vs. graphical thinking: i.e. the tendency for working with textual vs. dataflow programming environments. b) preference of self-made vs. pre-made tools. c) embodied vs. disembodied emphasis in playing and making instruments or compositions d) whether the person is a "techie" vs. "non-techie" where we tried to extract the level of people's "computer-literacy" and programming skills, e) academic vs. non-academic. We were interested in the question of how these audio programming environments (that mostly have their origins in academia) have filtered out into the mainstream culture.

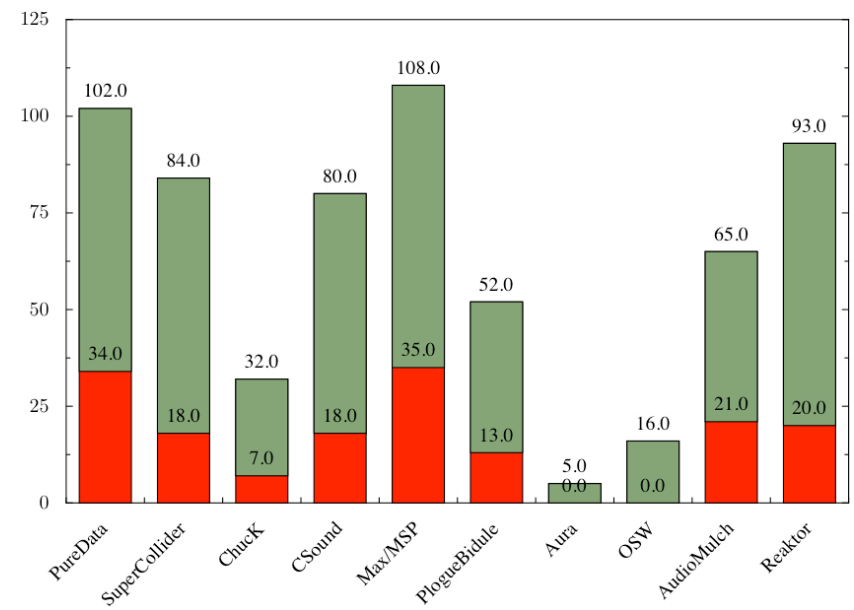

Figure 2. Tool-usage of the survey participants. The higher number shows how many people use or have used the specific tool. The lower number shows their tool of choice.

The continuum we used for marking this had values from 1 to 5 with "nil" as a valid entry where it was impossible to extract any meaningful value from the answers. In order to test how reliable this subjective method of categorising the answers was, we selected five random replies and gave the same set of replies to five different people to analyse. The comparison of the different analyses came out positively. The results were almost identical, with some minor differences on the left or right side of the continuum; never opposite interpretations.

\section{THE EVALUATION}

There were many questions in our survey that addressed the issues of acoustic vs. digital instruments from different angles but they were varied approaches or "interfaces" to some underlying topics of interest. In the next sections we will look at some selected topics and what we learned from the answers.

\subsection{The survey participant}

From analysing the demographic of the people answering the survey, we could divide the typical survey participant into two groups of which more than 90 percent of the participants would belong: a) People that have had over 20 years of studying music and playing acoustic instruments, therefore typically 30-40 years of age or older. They have been using the computer for their music for at least 10 years and usually have some form of programming experience. Many write their own software or use the common audio programming environments available today.
This group has thought a lot about their instruments and why they have chosen to work on their music using the computer. b) The other group consists of younger people that grew up with the computer and are also highly computer literate. Many of them have not studied music formally or practised an acoustic instrument but are using the computer as their instrument or environment for creating music. Here, of course, one could view all the time in front of the computer screen performing any task as part of the musical training. Naturally there was some degree of overlap between the two groups.

It might be illustrative to look at which operating systems the participants are running their tools on, and here we see that 45 people use Linux/GNU; 105 use Windows; and 88 use Mac OS. Of these 16 stated that they use both Mac OS and Linux/GNU; 30 use both Mac OS and Windows; 25 Linux/GNU and Windows; and 7 used all three. Other operating systems in use were NeXTstep, BSD and Solaris, one person each system.

\subsection{Acoustic vs. digital instruments}

The question we were concerned with here is how people experience the different qualities of acoustic instruments and digital instruments or software tools. Apart from the experiential and perceptual differences, do people think that the tools enframe or influence their work?

Many people found that an important difference in these two types of instruments lies in the fact that the digital instrument can be created for specific needs whereas the player has to "mould oneself" to the acoustic instrument. As the composed digital instrument can be very work specific, it lacks the generality of acoustic instruments. Related to this, some people reported discontent with the uncertainty of the continuation of commercial digital instruments or software environments. Their production could be discontinued or not supported on new operating systems. Unless open source is used, the proprietary protocols could become unsupported rendering the instruments objects of archaeology. In this regard, acoustic instruments have longer lifetime, which makes practising them more likely a continuous path to mastery.

Some survey participants expressed the wish for more limited expressive software instruments, i.e. not a software that tries to do it all but "does one thing well and not one hundred things badly". They would like to see software that has an easy learning curve but incorporates deep potential for further explorations, in order not to become bored with the instrument. True to form, the people asking for such software tools had a relatively long history as instrumentalists.

Some participants expressed how they found their time better spent working with digital technology, creating music or "experimenting with sound" rather than practising an acoustic instrument. Conversely, others talked about the dangers of getting side-tracked when using the computer, constantly looking for updates, reading mailing lists, testing other people's patches or instruments, even ending up browsing the web whilst trying to make music. Some talked about the "frightening blank space" of the audio programming patcher (meaning the endless possibilities) and found retreat in limited tools or acoustic instruments, whereas others were frustrated with the expressive limitations of the acoustic instruments and craved for more freedom and open work environments. Naturally, this went hand in hand with people's use of environments such as SuperCollider, ChucK, Pure Data and 
Max/MSP vs. preference of less open or more directive software like ProTools, Cubase, GarageBand, Fruityloops, etc.

Another issue of concern was latency. An acoustic instrument does not have latency as such, although in some cases there is a delay between the energy applied and the sounding result. In digital instruments there might be up to $50 \mathrm{~ms}$ latency that people put up with when playing a hardware controller; many seconds latency in networked performances; but also the organisational latency when opening patches, changing effect settings or in livecoding where one has to type a whole function before hearing the result (typically by hitting the Enter button). This artificial latency is characteristic of digital instruments, but not necessarily a negative property apart from the situation of using hardware controllers. However, the state of affairs in that field have improved drastically in recent years.

Table 1. Frequent comments on the positive and negative aspects of acoustic instruments.

\begin{tabular}{|l|l|}
\hline \multicolumn{1}{|c|}{ Acoustic - Positive } & \multicolumn{1}{c|}{ Acoustic - Negative } \\
\hline Tactile feedback & Lacking in range \\
Limitations inspiring & No editing out of mistakes \\
Traditions and legacy & No memory or intelligence \\
Musician reaches depth & Prone to cliché playing \\
Instrument becomes $2^{\text {nd }}$ nature & Too much tradition/history \\
Each instrument is unique & No experimentation in design \\
No latency & Inflexible - no dialog \\
Easier to express mood & Less microtonality or tunings \\
Extrovert state when playing & No inharmonic spectra \\
\hline
\end{tabular}

The question of originality came up frequently. People found it possible to be more original using the composed, digital instruments, precisely because of the lack of history and traditions. As one survey participant put it: "when playing an acoustic instrument, you are constantly referring to scales, styles, conventions, traditions and clichés that the instrument and the culture around it imposes on you. A musician can just play those conventions in autopilot without having to THINK at all. It's easy and unchallenging". This, of course, is a double-edged sword, as it is difficult in a live performance using software tools to refer to the musical reservoir in the spur of the moment. All such decisions have to be pre-programmed and thus pre-planned. This issue of originality also points to the limited scopes of some commercial software environments where the users are almost led into producing music of certain styles.

Last but not least, people were concerned with the arbitrary mappings in digital instruments.[4] There are no "natural" mappings between the exertion of bodily energy and the resulting sound. One participant described digital instruments as "more of a mind/brain endeavour." He continued and stated that "it is more difficult to remove the brain and become one with the physical embodiment of performing". Others talked about the perception of making the physical object vibrate and feeling the source of the sound in direct and natural ways being something that the computer systems lack with their buttons and sliders, soundcards and cables going out to the remote speakers. Yet another participant talked about the enriching experience of learning the vocabulary and voice of an instrument like the viola to its finest details, whereas with computer technology the voice is too broad to get to know it thoroughly.

\subsection{Affordances and Constraints}

Here we were interested in the question whether people relate differently to the affordances and the limitations of their acoustic and digital instruments.

It was a common agreement that the limitations of acoustic instruments were a source of inspiration and creativity. People talked about "pushing the boundaries" of the instrument and exploring its limits. Many participants said the same about digital instruments, but more commonly people were critical of the limitations of software. People felt that software limitations are due to engineering or software design, as opposed to the physical limitations of natural material like wood or strings. This fact makes people more critical of software tools than they are with acoustic instruments. There could be many reasons for this; one being that musical software is such a new field and naturally experimental whereas acoustic instruments have had centuries of refinement. Another observation that our data supports as well is that people normally start to learn an acoustic instrument at a very young age when things are more likely to be taken for granted. People see it as their fault if they cannot play the instrument properly, not the imperfection of the instrument design itself. This is different with digital instruments - at least with our survey participants - where people are more likely to criticise and see the limitations as weakness of the design rather than their own work methods or understanding of the system.

Table 2. Frequent comments on the positive and negative aspects of digital instruments.

\begin{tabular}{|l|l|}
\hline \multicolumn{1}{|c|}{ Digital - Positive } & \multicolumn{1}{c|}{ Digital - Negative } \\
\hline Free from musical traditions & Lacking in substance \\
Experimental - explorative & No legacy or continuation \\
Any sound and any interface & No haptic feedback \\
Designed for specific needs & Lacking social conventions \\
Freedom in mapping & Latency frequently a problem \\
Automation, intelligence & Disembodied experience \\
Good for composing with & Slave of the historical/acoustic \\
Easier to get into & Imitation of the acoustic \\
Not as limited to tonal music & Introvert state when playing \\
\hline
\end{tabular}

Most of the skilled instrumentalists saw the limitations of their acoustic instruments with positive eyes and viewed the potential both discovered and undiscovered - of the instrument as an expressive space in which they felt comfortable. People usually had an "emotional" affection towards their acoustic instrument (one of our questions asked about this) and they bonded with its character. This issue is very different in regard to people's feelings about their digital instruments. Survey participants often expressed frustrations with the technology, irritating limitations of software environments and dissatisfaction with how hardware needs constant upgrading, fixing and, not surprisingly, the use of electricity. One responder talked about how the limitations of acoustic instruments change or evolve constantly according to skill levels but also state of mood, whereas the limitations of software, once it has been learned and understood, are the limitations of the design. As another participant put it: "the creative challenge [in digital instruments] is to select and refine rather than expand".

In general people felt that the main power of digital instruments is that one can design the instrument for specific needs. The process of designing the instrument becomes a process of composing at 
the same time. The fact that people talk about "composing instruments" [8] yields a clear distinction from the acoustic world where instruments tend to be more general in order to play more varied pieces. This also explains why we do not see the continuity of digital instruments or interfaces through time: each instrument tends to be made for a specific and not general purpose. The power to be able to store conceptual structures in the tool itself renders it more specific and unique for a certain musical piece or performance and less adaptive for other situations. However, there is a continuum where instruments are on the one side unique and specific and on the other side general and multi-purpose. Creating a digital instrument always involves decisions on where to place the instrument on that continuum.

\subsection{The instrument maker criticised}

As discussed above, our survey shows that people have a different critical stance to the makers of acoustic and digital instruments (or software). This is reflected in the way people relate to the instruments themselves. The fact that acoustic instruments seem to have existed forever (and the survey shows that the majority of people do not have a very thorough historical knowledge of their instrument) makes people less likely to step back and actively criticise their instrument of choice.

Almost all the participants stated that their acoustic instruments have been built from ergonomic and aesthetic/timbral considerations and saw the evolution of their instrument as a refinement where it is moulded to the human body. There is, however, evidence that orchestral instruments were developed primarily with the view to stabilise intonation and augment acoustic power or loudness.[5] In fact, the young but strong tradition of digital music instruments and interface building is perhaps more consciously concerned with ergonomics and human-tool interaction than we find in the history of acoustic instrument building. Ergonomics have at least become more prominent in the way people think when building their musical tools. An agreed view was that the difficulty of building masterly interfaces in the digital realm is largely because of the complexity of the medium and the unnatural or arbitrary nature of its input and output mappings.

In Being and Time [3], the philosopher Martin Heidegger talks about two cognitive or phenomenological modalities in the way we look at the world. There is on the one hand the usage of tools, where the tools are ready-at-hand and are applied in a finely orchestrated way by the trained body, and, on the other hand, the moment when the tool breaks and it becomes present-at-hand, i.e. the user of the tool has to actively think what is wrong with the tool, and look at its mechanism from a new and critical stance. Heidegger takes the example of a carpenter who uses the hammer day in and day out without thinking about it. Only when the head falls off the hammer will the carpenter look at the hammer from a different perspective and see it in its true phenomenological light. As digital instruments/software are normally applied on the computer, we are more likely to have these phenomenological breaks. The tool becomes present-at-hand and we have to actively think about why something is not working or what would be the best way of applying a specific tool for a certain task. In fact, the way we use a computer and digital instruments is a constant oscillation between these two modes of being ready-at-hand and present-at-hand. We forget ourselves in working with the tool for a while, but suddenly we have to open or save a file, retrieve a stored setting, switch between plug-ins or instruments, zoom into some details, open a new window, shake the mouse to find the cursor, plug in the power cable when the battery is low, kill a chat client when a "buddy" suddenly calls in the middle of a session, etc. In this respect, many of the participants saw the computer as a distracting tool that did not lend itself to deep concentration.

\subsection{Entropy and control in instruments}

Here we were interested to know how people relate to the nondeterministic nature of their instruments and if it differs whether the instrument is acoustic or digital.

We had two trends of responses here. It was mostly agreed that the accidental or the entropic in acoustic instruments could be a source of joy and inspiration. Some people talked about playing with the tension of going out on the "slippery ice" where there was less control of the instrument. Typically people did not have the same view of digital instruments. When they go wrong or unpredictable, it is usually because of a bug or a fault in the way they are set up and most people did not like that. However, there was a strand of people that enjoyed and actively searched for such "glitches" in software, which of course has resulted in the well known musical style called "Glitch".

That said, according to our data, the process of exploration is a very common way of working with software, where people set up a system in the form of a space of sonic parameters where the user navigates that space until a desired sound or musical pattern is found. ${ }^{3}$ This style of working is quite common in generative music and in computationally creative software where artificial intelligence is used to generate the material and the final fitness function of the system tends to be the aesthetic judgement of the user.

\subsection{Time and embodiment}

As most people would have guessed, we found that the time spent playing an instrument emphasised the desire to have physical control and use the body in a musical performance. When analysing the embodied-disembodied continuous scale we interpreted people's answers into, we saw that the longer people had played an acoustic instrument, the more they stressed the importance of embodiment in their musical practise. Playing digital instruments seems to be less of an embodied practise (where motor-memory has been established) as the mapping between gesture and sound can be changed so easily by changing a variable, a setting, a patch or a program. Some responders noted that working with digital instruments or software systems had forced them to re-evaluate the way they understand and play their acoustic instrument. Of course, the contrary has to be true as well.

There are a few things to note here. Most of the people that answered the survey were both acoustic and digital instrumentalists and were confident with the qualities of both worlds. It seems that people subscribe positively to the qualities of each of the two instrumental modalities - acoustic and digital and do not try to impose working patterns that work in one type instrument onto the other. In general people seem to approach each instrument on its own merits and choose to spend time on it if it gives them some challenge or excitement.

\footnotetext{
${ }^{3}$ For a discussion of compositional processes in electronic music, see Eaglestone, Upton and Ford [2]
} 


\section{INTERESTING COMMENTS}

There were some comments that are worth printing here due to their direct and clear presentation:

"I don't feel like I'm playing a digital instrument so much as operating it."

"Eternal upgrading makes me nervous."

"full control is not interesting for experimentation. but lack of control is not useful for composition."

"Can a software "instrument" really be considered an instrument, or is it something radically different?"

"The relationship with my first instrument (guitar) is a love / hate one, as over the years I developed a lot of musical habits that are hard to get rid of ;-)"

“j'entretiens un certain rapport avec mes machines. Impossible pour moi de penser à revendre une machine."

"I think acoustic instruments tend to be more right brain or spatial, and digital instruments tend to be more left brain and linguistic."

\section{DISCUSSION}

There were many surprising and interesting findings that came out of this survey. First of all, we were surprised by the high mean age of the survey participants. We wondered if the reason for the high average age could be the nature of the questions, especially considering the questions regarding embodiment. Perhaps the questions are not as relevant to the younger people who have been brought up with the computer and are less alienated by the different modes of physical vs. virtual interaction? A likely explanation is that the mean age of the survey participants is reflecting that of the members of the mailing lists we posted the survey to.

It is illustrative that the majority of people answering the survey were involved with academia or had an academic or conservatory education. This helps to explain the high mean age but also the high level of analysis that most people had applied to their tools. We noted that the time spent playing an instrument increases the focus on embodiment in players and as such the questions of this survey might have connected better with the older musicians.

An important point to raise here is that whereas the survey focused on the differences of acoustic and digital instruments and people's perception of those, the fact is that most people are content with working with both instrumental modalities and subscribe to the different qualities of each when using those specific tools. Many of the people answering the survey used the computer in combination with acoustic instruments, especially for things that the computer excels at such as musical analysis, adaptive effects, hyper-instruments and artificial intelligence.

A clear polarity between the acoustic and digital instruments is the division between an instrument maker and a musician/composer in acoustic instruments. In the field of digital instruments, designing an instrument often overlaps with the musical composition itself (or at least designing its conditions). There is a continuum where people's work can be placed: from a personal expression in the form of a composition to a software that can be distributed for others to use.

Another interesting trait we noticed in the survey was the question of open source software. Many people are using Linux or expressing desire to do so because they feel that they have more control over things and are less directed by some commercial company's ideas of how to set up the working environment or compose/perform music. The questions of open protocols and standards, of legacy in software, of collaborative design and freedom to change the system were all important issues here.

\section{FUTURE WORK}

The topics of this survey revealed many more questions that would have been unconceivable without the process of making this survey and reading the replies. The next step in our research will be to perform interview sessions with both acoustic and electronic musicians and laypeople. We would like to find out how people experience graphical user interface design in musical software and whether they think the functionality of the software represents or fits the mental model they already have about how to compose and/or perform music. Can software be seen as epistemic tools where acoustic instruments are more pragmatic tools?

\section{ACKNOWLEDGMENTS}

Working on this survey has been immensely interesting and we would like to thank all the participants for their efforts in answering the survey. Some of the answers were profoundly intriguing, some gave nice twists in perspective and some were incredibly witty. We would like to thank Nick Collins, Marcelo Wanderley, Chris Thornton, Greg Hooper, Cian O'Connor, Tom Hall, Heimir Snorrason, Leire Vergara, Birta Thrastardottir, Monica Guerrero and all the other people that were involved in helping us with the survey.

\section{REFERENCES}

[1] Duignan, Matthew; Noble, James \& Biddle, Robert. "A Taxonomy of Sequencer User-Interfaces" in Proceedings of ICMC 2005, Barcelona: Escola Superior de Música de Catalunya, 2005.

[2] Eaglestone, Barry; Upton, Catherine \& Ford, Nigel. "The Compositional Process of Electroacoustic Composers: Contrasting Perspectives" in Proceedings of ICMC 2005, Barcelona: Escola Superior de Música de Catalunya, 2005.

[3] Heidegger, Martin. Being and Time. Oxford: Blackwell Publishers, 1995. Pp. 102-122.

[4] Hunt, Andy; Wanderley, Marcelo M. \& Paradis, Matthew. "The Importance of Parameter Mapping in Electronic Instrument Design" in Proceedings of NIME 2002. Limerick: University of Limerick, Department of Computer Science and Information Systems, 2002

[5] Jordá, Sergi. Digital Lutherie: Crafting musical computers for new music's performance and improvisation. $\mathrm{PhD}$ thesis. Barcelona: Universitat Pompeu Fabra, 2005. p. 169.

[6] Magnusson, Thor. "ixi software: Open Controllers for Open Source Software" in Proceedings of ICMC 2005. Barcelona: Escola Superior de Música de Catalunya, 2005.

[7] Magnusson, Thor. "Screen-Based Musical Instruments as Semiotic-Machines" in Proceedings of NIME 2006. Paris: IRCAM, 2006.

[8] Momeni, Ali. "Composing instruments: Inventing and performing with generative computer-based instruments", PhD Dissertation, UC Berkeley. 2005. 